\title{
Exact solutions for mass-dependent irreversible aggregations
}

\author{
Seung-Woo Son, ${ }^{1}$ Claire Christensen, ${ }^{1}$ Golnoosh Bizhani, ${ }^{1}$ Peter Grassberger, ${ }^{1,2}$ and Maya Paczuski ${ }^{1}$ \\ ${ }^{1}$ Complexity Science Group, University of Calgary, Calgary T2N 1N4, Canada \\ ${ }^{2}$ Forschungszentrum Jülich, D-52425 Jülich, Germany
}

(Received 31 August 2011; published 31 October 2011)

\begin{abstract}
We consider the mass-dependent aggregation process $(k+1) X \rightarrow X$, given a fixed number of unit mass particles in the initial state. One cluster is chosen proportional to its mass and is merged into one, either with $k$ neighbors in one dimension, or-in the well-mixed case — with $k$ other clusters picked randomly. We find the same combinatorial exact solutions for the probability to find any given configuration of particles on a ring or line, and in the well-mixed case. The mass distribution of a single cluster exhibits scaling laws and the finite-size scaling form is given. The relation to the classical sum kernel of irreversible aggregation is discussed.
\end{abstract}

DOI: 10.1103/PhysRevE.84.040102

PACS number(s): 05.70.Ln, 89.75.Da, 89.75.Hc

Recently the theory of irreversible aggregation was revisited in view of renormalization of complex networks [1]. In Ref. [1], a simple mapping between random sequential renormalization (RSR) [2,3] and irreversible aggregation [4] was pointed out, where a local random renormalization step to produce a new "supernode" in complex networks corresponds to one aggregation event of "molecules." Exact combinatorial analyses, both in one dimension (without diffusion) and in the well-mixed case, gave the same scaling law of cluster mass distribution. The corresponding exponent only depends on $k$, the number of interacting neighbors [1]. This RSR procedure corresponds to the "constant" kernel of irreversible aggregation (one of three well-known "classical" kernels-constant, sum, and product kernels [4]). In this Rapid Communication, we show the relation between mass-dependent RSR and irreversible aggregation with the sum kernel. Applying the same combinatorial technique of Ref. [1], we find the exact solutions for mass-dependent irreversible aggregation as well.

Here we consider models governed by the reaction $(k+1) X \rightarrow X$, where a cluster is picked randomly, in proportion to its mass, after which it coalesces with $k$ other clusters. In the case of one-dimensional models these are $k$ neighbors, while they are $k$ other clusters chosen randomly in the case of well-mixed systems. In both cases, the other clusters are chosen independent of their masses. The mass of the newly formed cluster is the sum of the $(k+1)$ masses. For one-dimensional models, both a ring with periodic boundary condition and a line with open boundary condition are considered. Reactions are allowed only if there is a sufficient number $k$ of available clusters.

First, let us consider the model defined on a "ring." Initially, $N_{0}$ particles of unit mass $(m=1)$ are placed on ringlike beads (see Fig. 1). Each particle is labeled by $i \in\left[1, \ldots, N_{0}\right]$. At each time, one cluster is picked in proportion to its mass, and is subsequently merged with its $k$ right neighbors into one big cluster having a mass equal to the sum of the $(k+1)$ masses. Cluster masses are therefore restricted to $m \equiv 1(\bmod k)$. This can be written as $m-1=k s$, where $s$ is the number of aggregation events needed to make a cluster of mass $m$. Similarly the number of clusters at any time $t$ is given by $N=N_{0}-k t$, where time $t$ is denoted by positive integers representing the total number of aggregation events. We do not allow two events to happen simultaneously in this study. Otherwise they can happen either at regular intervals, intermittently, or according to a Poisson process.

To find the probability that any of the $N$ clusters picked at random has mass $m$ resulting from $s$ aggregation events, we follow an approach similar to the one introduced in Ref. [1]. The crucial observation that makes the analysis simple is that picking clusters according to their mass is equivalent to picking sites with uniform probability, since a cluster of mass $m$ occupies $m$ sites. Let $i$ be any site (e.g., $i=1$ ), and let $\pi_{N}^{N_{0}}(m)$ be the probability that a cluster of mass $m$ starts at this site and occupies the sites $(i, i+1, \ldots, i+m-1)$. The probability that any of the $N$ clusters picked at random has mass $m$ after $t$ events is then

$$
p_{N}^{N_{0}}(m)=\frac{N_{0}}{N} \pi_{N}^{N_{0}}(m)
$$

and

$$
\pi_{N}^{N_{0}}(m)=\left(\begin{array}{c}
t \\
s
\end{array}\right) \frac{n_{\text {cluster }} n_{\text {rest }}}{n_{\text {total }}},
$$

where $n_{\text {cluster }}$ is the number of possible histories of aggregation events $\left(i_{1}, i_{2}, \ldots, i_{s}\right)$ leading to a cluster of mass $m, n_{\text {rest }}$ is the number of possible ways to form the other $(N-1)$ clusters, and $n_{\text {total }}$ is the total number of histories for $t$ merging events. The binomial coefficient $\left(\begin{array}{l}t \\ s\end{array}\right)$ corresponds to the number of choices associated with different time orderings for the $s$ events in the cluster of mass $m$ and the $(t-s)$ events in the rest of the clusters.

The total number of all histories involving $t$ events is simply

$$
n_{\text {total }}=N_{0}^{t} \text {. }
$$

This is to be contrasted to the number of histories $n_{N}^{\left[1, N_{0}\right]}$ that lead to the first cluster starting at $i=1$ and the $N$ th ending at $N_{0}$. A somewhat more involved argument gives

$$
n_{N}^{\left[1, N_{0}\right]}=N N_{0}^{t-1} \text {. }
$$

The number of histories leading to a single cluster of mass $m$ covering the sites of interval $[1, m]$ is thus

$$
n_{\text {cluster }}=n_{1}^{[1, m]}=m^{s-1},
$$

while

$$
n_{\text {rest }}=n_{N-1}^{\left[1, N_{0}-m\right]}=(N-1)\left(N_{0}-m\right)^{t-s-1} .
$$




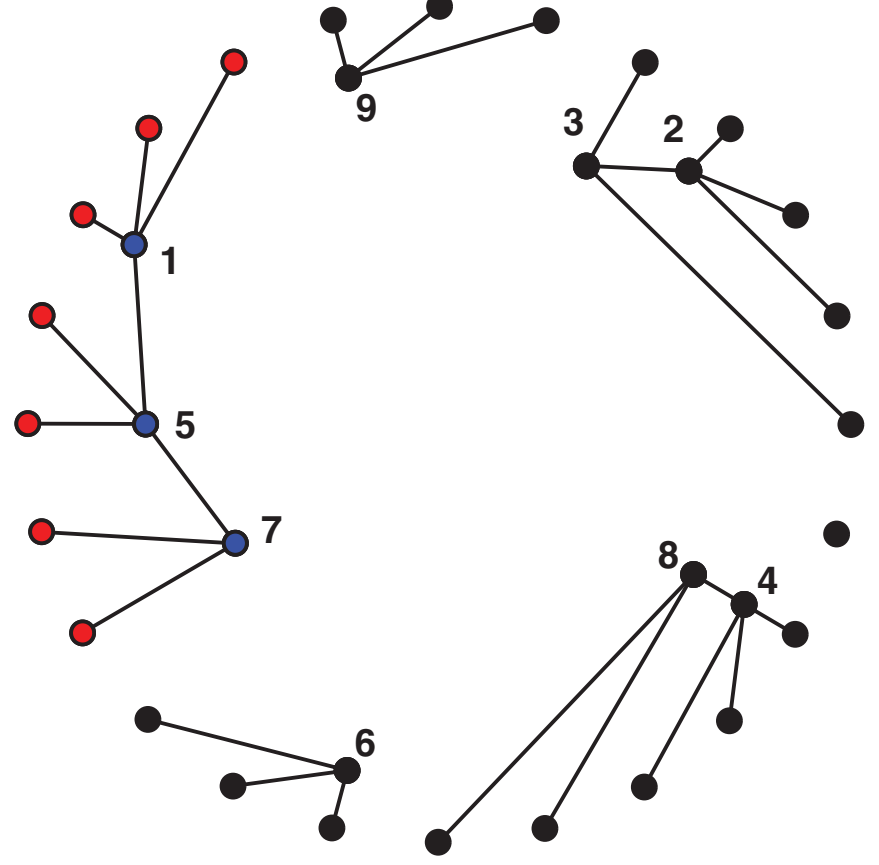

FIG. 1. (Color online) Illustration of aggregation on a ring with $k=2, N_{0}=24$, and $N=6$. The tree in color corresponds to a cluster of mass $m=7$. It has seven leaves (red/light gray) and three internal nodes (blue/dark gray). The numbers beside internal nodes correspond to the time when coalescence occurs.

Combining Eqs. (1)-(6), we finally obtain

$$
p_{N}^{N_{0}}(m)=\frac{N-1}{N}\left(\begin{array}{l}
t \\
s
\end{array}\right) \frac{m^{s-1}\left(N_{0}-m\right)^{t-s-1}}{N_{0}^{t-1}} .
$$

For this mass-dependent aggregation process, we can also work out the joint probability distributions for masses of adjacent clusters. We denote by $p_{N}^{N_{0}}\left(m_{1}, m_{2}\right)$ the probability to find a cluster of mass $m_{1}$ followed immediately to the right by a cluster of mass $m_{2}$. This is nonzero only if $m_{1}=k s_{1}+1$ and $m_{2}=k s_{2}+1$, where $s_{\alpha}$ is the number of aggregation events needed to form a cluster of mass $m_{\alpha}$. By the previous arguments, we get

$$
p_{N}^{N_{0}}\left(m_{1}, m_{2}\right)=\frac{N-2}{N}\left(\begin{array}{c}
t \\
s_{0}, s_{1}, s_{2}
\end{array}\right) \frac{m_{1}^{s_{1}-1} m_{2}^{s_{2}-1} m_{0}^{s_{0}-1}}{N_{0}^{t-1}}
$$

where $s_{0}=t-\sum_{\beta=1}^{\alpha} s_{\beta}$ and $m_{0}=N_{0}-\sum_{\beta=1}^{\alpha} m_{\beta}$. It is interesting to note that this joint probability properly holds the following relation:

$$
p_{N}^{N_{0}}\left(m_{1}, m_{2}\right)=p_{N}^{N_{0}}\left(m_{1}\right) p_{N-1}^{N_{0}-m_{1}}\left(m_{2}\right) .
$$

For any $1 \leqslant \alpha \leqslant N-1$, the joint probability distribution for $\alpha$ consecutive adjacent clusters is given by

$p_{N}^{N_{0}}\left(m_{1}, \ldots, m_{\alpha}\right)=\frac{N-\alpha}{N} \frac{\mathcal{T}[t,\{s\}, \alpha+1] \prod_{\beta=0}^{\alpha} m_{\beta}^{s_{\beta}-1}}{N_{0}^{t-1}}$,

where we used the multinomial coefficient

$$
\mathcal{T}[t,\{s\}, \alpha+1]=\left(\begin{array}{c}
t \\
s_{0}, \ldots, s_{\alpha}
\end{array}\right) .
$$

In particular, this can be done for the joint distribution for all $N$ masses by setting $\alpha=N-1$. The resulting expression is always invariant under any permutations of $N$ numbers $\left(m_{1}, \ldots, m_{N}\right)$, as was the case with mass-independent aggregation [1]. Hence the $N$-cluster probability is independent of the spatial ordering of the clusters. Therefore, there are no spatial correlations, even though there are obvious correlations between the masses at any given time. For this reason [and as verified in detail using Eq. (4) instead of Eq. (3)], the joint probability for $N$ masses on a line, i.e., a one-dimensional system with open boundaries, is also given as Eq. (8), showing that the two models lead to the same statistics for any $\alpha$.

The absence of spatial correlations indicates that the same dynamics might also result from the well-mixed case. To check this, we now start with a bucket containing $N_{0}$ balls, each of unit mass. An event consists of first picking one ball with probability proportional to its mass and then choosing $k$ balls out of the bucket, independent of their masses. The balls are merged and a new ball, having a mass equal the sum of the masses of its $(k+1)$ constituents, is returned to the bucket. This process repeats until $N$ clusters remain.

The single-cluster mass distribution for the well-mixed model can be obtained using the same strategy as before. Since events now correspond to choosing one ball with a mass-weighted probability, and $k$ balls out of $\left(N_{0}-k t-1\right)$ balls randomly, we have a $t$ power of $N_{0}$ and a product of binomial coefficients

$$
\begin{aligned}
n_{\text {total }} & =N_{0}\left(\begin{array}{c}
N_{0}-1 \\
k
\end{array}\right) \cdots N_{0}\left(\begin{array}{c}
N+k-1 \\
k
\end{array}\right) \\
& =\frac{N_{0}^{t}}{(k !)^{t}} \frac{\left(N_{0}-1\right) !}{(N-1) !}=\frac{N_{0}^{t-1}}{(k !)^{t}} \frac{N_{0} !}{(N-1) !} .
\end{aligned}
$$

The expressions for $n_{\text {cluster }}$ and $n_{\text {rest }}$ are analogously

$$
\begin{gathered}
n_{\text {cluster }}=\frac{m^{s-1}}{(k !)^{s}} m ! \\
n_{\text {rest }}=\frac{\left(N_{0}-m\right)^{t-s-1}}{(k !)^{t-s}} \frac{\left(N_{0}-m\right) !}{(N-2) !} .
\end{gathered}
$$

The number of time orderings is exactly the same as before, but the first factor $N_{0} / N$ in Eq. (1) has to be replaced by $\frac{1}{N}\left(\begin{array}{c}N_{0} \\ m\end{array}\right)$. Putting all these considerations together, many cancellations take place, leading exactly to Eq. (7) again. This argument can be similarly extended to get the full $N$-particle distribution function, obtaining exactly the same result as Eq. (8) for any $k$ and $\alpha$.

Let us look at the characteristics of the solutions. Even though the composition principle is the same as that of mass-independent aggregation in Ref. [1], the final solution and the characteristics are quite different. First, cluster size distributions at several different times are shown in Fig. 2 for $N_{0}=100$ and $k=2$. The symbols indicate the numerical simulation results over $10^{6}$ realizations and the solid lines are the exact solutions of Eq. (7). The tail region corresponding to large cluster sizes changes from a fast exponential decay to an increasing power law as the merging process approaches termination. The turning point is at $N \sim \sqrt{N_{0}}$. When $N=2$, since the sum of the two cluster sizes is always $N_{0}$, the distribution $p_{N}^{N_{0}}(m)$ is symmetric under the exchange 


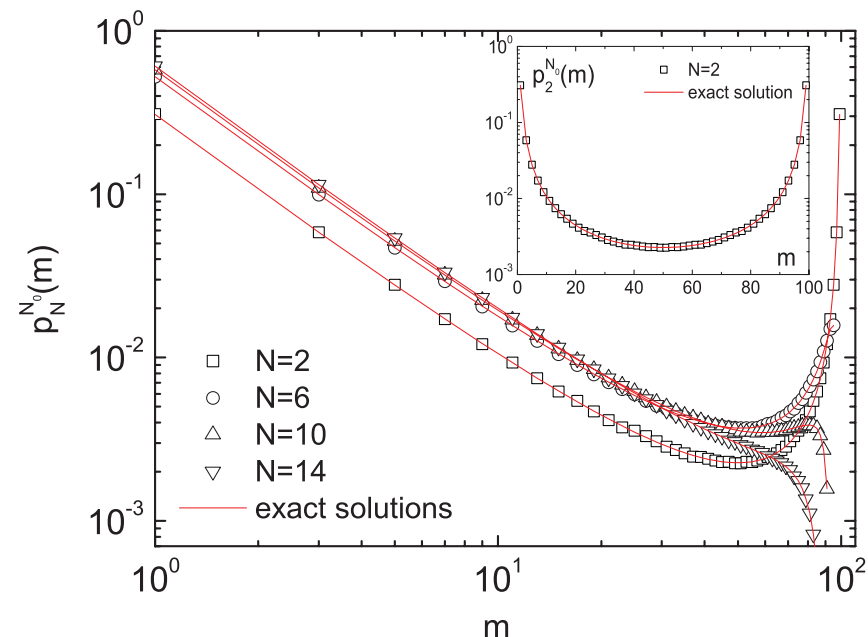

FIG. 2. (Color online) Cluster size distributions after $t=49$ $(N=2)$ events for $k=2$, for different values of $N$ averaged over $10^{6}$ realizations compared to exact results. The power-law slope of small $m$ is $-3 / 2$ independent of $k$. The large size behavior changes from an increasing power law to a decreasing one at $N \sim \sqrt{N_{0}}$. The inset shows the symmetric distribution for $N=2$.

$m \leftrightarrow N_{0}-m$ for any $k$. The symmetric distribution for $N=2$ is shown in the inset of the Fig. 2.

When $N_{0} \rightarrow \infty$, asymptotic power laws can be determined using Stirling's formula. If $N$ is fixed and both $m$ and $\left(N_{0}-m\right) \rightarrow \infty$, one obtains the scaling form

$$
\begin{aligned}
p_{N}^{N_{0}}(m) & \sim N_{0}^{-\frac{3}{2}}\left[\frac{m}{N_{0}}\left(1-\frac{m}{N_{0}}\right)\right]^{-\frac{3}{2}} e^{-\frac{N^{2}}{k N_{0}} \frac{m}{N_{0}}\left(1-\frac{m}{N_{0}}\right)^{-1}} \\
& \sim N_{0}^{-\frac{3}{2}} f\left(\frac{m}{N_{0}}, \frac{N}{\sqrt{N_{0}}}\right) .
\end{aligned}
$$

For small masses, this gives a decreasing power law, with exponent $-3 / 2$, independent of $k$. Interestingly, this is very different from mass-independent aggregation, for which the analogous exponent depends on $k$ and is equal to $-1+1 / k[1]$. The exponent $-3 / 2$ is the same as that for the aggregation with the sum kernel of the irreversible aggregation obtained in Ref. [5]. Indeed, the rate equation for the current aggregation model in mean-field theory is the same as for the sum kernel $[4,5]$. For the $k=1$ case, the rate equation is simply

$$
\begin{aligned}
\Delta p_{m} & =\sum_{m^{\prime}=1}^{m} m^{\prime} p_{m^{\prime}} p_{m-m^{\prime}}-p_{m} \sum_{m^{\prime}=1}^{\infty}\left(m+m^{\prime}\right) p_{m^{\prime}} \\
& =\frac{m}{2} \sum_{m^{\prime}=1}^{m} p_{m^{\prime}} p_{m-m^{\prime}}-(m+\bar{m}) p_{m},
\end{aligned}
$$

where $p_{m}$ denotes $p_{N}^{N_{0}}(m)$ to make the equation more concise, and where $\bar{m}$ means the mean cluster size. Equation (13) is the same as the rate equation for the sum kernel in Refs. [4] and [5]. The behavior for large $m$ is different, however, and is not described by mean-field theory.

According to Eq. (12), $N_{0}^{\frac{3}{2}} p_{N}^{N_{0}}(m)$ should be a function of $m / N_{0}$ only for fixed

$$
y=\frac{N^{2}}{k N_{0}}
$$

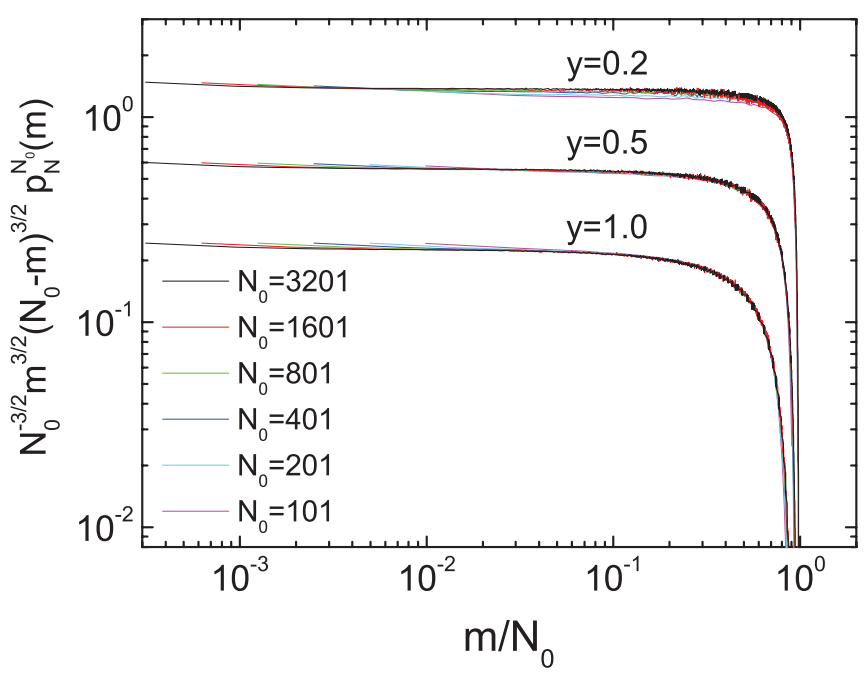

FIG. 3. (Color online) Finite-size scaling collapses for different $y$ and fixed $k=2$. Collapse lines for $y=0.2$ and $y=1.0$ are shifted up and down to make them distinguishable from other collapse lines.

and for $N \ll N_{0}, N_{0}-m$. The resulting data collapse is shown in Fig. 3, where we also factored out a power of $m / N_{0}$ to make the curves less steep. Notice that $N$ and $N_{0}$ are related by $N \equiv N_{0}(\bmod k)$, which implies that the values of $y$ used in this plot are not strictly constant but deviate slightly from their nominal values for small $N_{0}$, which causes the deviation from a perfect collapse for $y=0.2$. Even for different values of $k$, this scaling function works, as can be seen in Fig. 4, where the scaling collapses for three cases $k=1,2$, and 4, are shown. Surprisingly, this means that the process of choosing a cluster proportional to its mass in conjunction with choosing two clusters at random for $k=2$ is asymptotically the same as repeating the merging process for $k=1$ twice in the sense of the scaled mass.

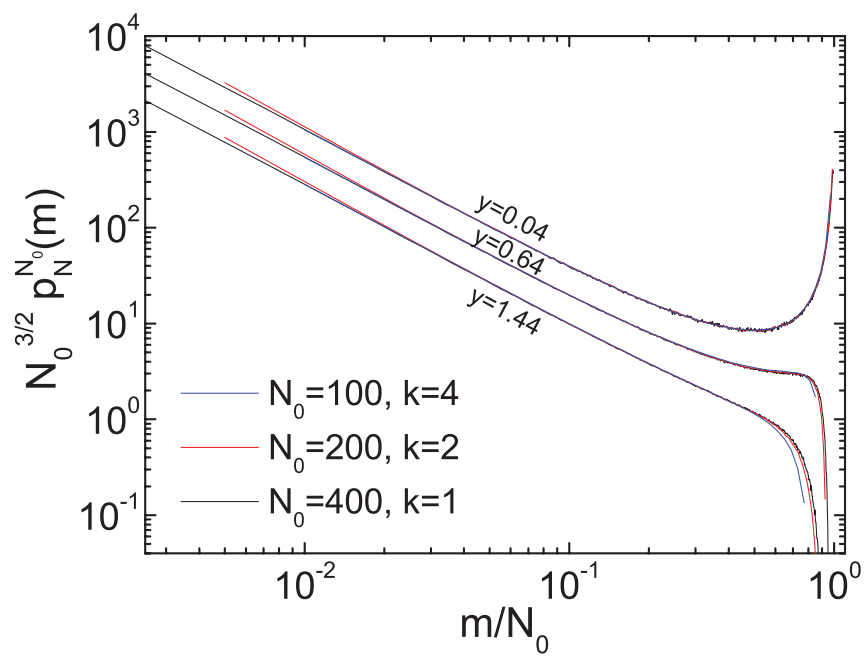

FIG. 4. (Color online) Finite-size scaling collapses for different $k$. In order to check the scaling collapses for different $k, k N_{0}$ is fixed at 400 and $N=4,16$, and 24, which correspond to $y=0.04,0.64$, and 1.44. Collapse lines for $y=0.04$ and $y=1.44$ are shifted up and down to make them distinguishable from each other. 
The probability $p_{N}^{N_{0}}(m)$ satisfies the following recursion relation:

$$
p_{N+k}^{N_{0}}(m)=A \sum_{m^{\prime}=m+k}^{N_{0}-N+1} \frac{m^{\prime}-1}{m^{\prime}} p_{N}^{N_{0}}\left(m^{\prime}\right) p_{k+1}^{m^{\prime}}(m),
$$

with

$$
A=\frac{N_{0} N(k+1)}{\left(N_{0}-N\right)(N+k)},
$$

where the prime on the summation symbol indicates that $m^{\prime}$ must increase in steps of $k$. Interestingly this quadratic recursion relation corresponds to the time-reversed process of aggregation, i.e., fragmentation. As with the quadratic recursion relation of mass-independent aggregation [1], the mass distribution at $N+k$ is given by the product of the mass distribution at $N$ describing the relative probabilities with which the cluster fragments, given by $p_{k+1}^{m^{\prime}}(m)$, and the total fragmentation probability. The latter was just $\propto\left(m^{\prime}-1\right)$ in the mass-independent case [1], while now it is proportional to $\frac{m^{\prime}-1}{m^{\prime}}$. Equation (15) follows then by considering how fragmentation leading to a cluster with mass $m$ goes through an intermediary with mass $m^{\prime}$.

We also examined numerically the aggregation processes where the clusters were chosen with probabilities proportional to higher powers of their mass, in particular, $\propto m^{2}$ and $\propto m^{3}$, i.e., the square of a cluster's mass and the cubic of a cluster's mass. The asymptotic power law exponents are roughly $-5 / 2$ and $-7 / 2$ for the $m^{2}$ dependence and $m^{3}$ dependence, respectively. However, to the best of our knowledge, exact solutions for these cases have not yet been found.

In summary, we derived the exact solutions for the probabilities to find any configuration after a fixed number of aggregation events in the models where a cluster picked with probability proportional to its mass aggregates with $k$ other particles. More specifically, we studied three versions of this process (particles on a ring joining with nearest neighbors, particles on an open-ended line, and the wellmixed case), and found exactly the same solutions using combinatorial counting. We attribute this to the absence of spatial correlations, although they are a priori not excluded. Differently from the mass-independent random sequential renormalization (RSR), which shows $k$-dependent exponents in scaling laws for small masses, the cluster size distribution follows a power law with exponent $-3 / 2$ independent of $k$, which is the same with that of the sum kernel for irreversible aggregation. Finally, the aggregation process is also related to a time-reversed fragmentation process, the characteristics of which are briefly discussed.

Mass-dependent RSR and the related aggregation process was also considered in two dimensions [3], where a runaway giant cluster exists after a few steps and takes all merging action. The behavior is very similar to the gelation in the aggregation process with the product kernel, but aggregation events in two-dimensional RSR involve fluctuating numbers of neighbors, differently from the aggregation process considered in the present Rapid Communication.
[1] S.-W. Son, G. Bizhani, C. Christensen, P. Grassberger, and M. Paczuski, Europhys. Lett. 95, 58007 (2011).

[2] G. Bizhani, V. Sood, M. Paczuski, and P. Grassberger, Phys. Rev. E 83, 036110 (2011).
[3] C. Christensen, G. Bizhani, S.-W. Son, M. Paczuski, and P. Grassberger, e-print arXiv:1012.1070.

[4] F. Leyvraz, Phys. Rep. 383, 95 (2003), and references therein.

[5] P. Krapivsky, J. Phys. A 24, 4697 (1991). 